\title{
Fenestration during Fontan palliation: Now the exception instead of the rule
}

\author{
Jorge D. Salazar, MD, ${ }^{\mathrm{a}, \mathrm{d}}$ Farhan Zafar, MD, ${ }^{\mathrm{d}}$ Kashif Siddiqui, MD, ${ }^{\mathrm{d}}$ Ryan D. Coleman, BA, ${ }^{\mathrm{d}}$ \\ David L. S. Morales, MD, ${ }^{\text {a,d }}$ Jeffrey S. Heinle, MD, ${ }^{\text {a,d }}$ Joseph W. Rossano, MD, ${ }^{\text {b,e }}$ \\ Emad B. Mossad, MD, ${ }^{\text {, f }}$ and Charles D. Fraser, Jr, MD ${ }^{\mathrm{a}, \mathrm{d}}$
}

\begin{abstract}
Objective: Fenestration during Fontan palliation has traditionally been used to decrease surgical morbidity and mortality, particularly in high-risk cases. Potential limitations include oxygen desaturation, risk of paradoxic embolism, and need for late intervention. Our practice has evolved away from routine fenestration with increased extracardiac conduit use. We reviewed our experience with Fontan palliation and retrospectively assessed outcomes with decreased fenestration.
\end{abstract}

Methods: Between January 2002 and April 2008, 226 patients underwent primary Fontan palliation. Outcomes were assessed by hospital stay, chest drain duration, short- and long-term survivals, and late interventions.

Results: Anatomic subtypes were single left ventricle $(n=88,38.9 \%)$, single right ventricle $(n=78,34.5 \%)$, common ventricle $(n=19,8.4 \%)$, and heterotaxy syndrome $(n=41,18.1 \%)$. Lateral tunnel connection was created in 69 patients (30.5\%); extracardiac connection was created in $157(69.5 \%)$. Mean age and weight at surgery were $4.3 \pm 3.8$ years and $17.2 \pm 9 \mathrm{~kg}$, respectively. In 2002, 14 of 16 patients $(87.5 \%)$ had fenestrated Fon$\tan$ circulations, versus 2 of $32(6.3 \%)$ in 2008. Mean hospital stay was $10.8 \pm 8.8$ days. Survival to discharge or 30 days was $98.7 \%$. There were $2(0.9 \%)$ late deaths during mean follow-up of $2.0 \pm 1.7$ years. Outcomes were equivalent between fenestrated and nonfenestrated procedures across anatomic subtypes.

Conclusions: Highly selective use of Fontan fenestration is achievable while maintaining excellent outcomes without increased surgical morbidity or mortality, irrespective of anatomic subtype. Risks of hypoxia, systemic embolism, and late instrumentation can be avoided in most cases. (J Thorac Cardiovasc Surg 2010;140:129-36)

Since first being introduced in 1971 by Dr Francis Fontan, the Fontan palliation for patients with single-ventricle anatomy has undergone significant evolution. ${ }^{1}$ From the atriopulmonary Fontan procedure first put forward for repair of tricuspid atresia, surgical approaches have transitioned to a total cavopulmonary anastomosis with an intracardiac lateral tunnel and, most recently, the extracardiac Fontan connection. ${ }^{2-5}$

Potential challenges encountered with Fontan physiology include low postoperative cardiac output, pleural and pericardial effusions, ascites, ventricular dysfunction, diminished exercise performance, and protein-losing enteropathy. ${ }^{6-8}$

\footnotetext{
From the Division of Congenital Heart Surgery, ${ }^{\text {a }}$ Department of Surgery, Section of Pediatric Cardiology, ${ }^{b}$ the Department of Pediatrics, and the Section of Pediatric Cardiovascular Anesthesia, ${ }^{c}$ Department of Anesthesiology, Baylor College of Medicine, Houston, Tex; and the Departments of Congenital Heart Surgery, ${ }^{d}$ Pediatric Cardiology, ${ }^{\mathrm{e}}$ and Pediatric Cardiovascular Anesthesia, ${ }^{\mathrm{f}}$ Texas Children's Hospital, Houston, Tex.

Disclosures: None.

Read at the Eighty-ninth Annual Meeting of The American Association for Thoracic Surgery, Boston, Mass, May 9-13, 2009.

Received for publication May 21, 2009; revisions received Feb 21, 2010; accepted for publication March 16, 2010.

Address for reprints: Jorge D. Salazar, MD, Division of Congenital Heart Surgery, Texas Children's Hospital, 6621 Fannin St MC-WT 19345H, Houston, TX 77030 (E-mail: jsalazar@umc.edu). $0022-5223 / \$ 36.00$

Copyright (c) 2010 Published by Elsevier Inc. on behalf of The American Association for Thoracic Surgery

doi:10.1016/j.jtcvs.2010.03.013
}

Routine fenestration of the Fontan circuit has been used to combat these morbidities and to improve operative survival. ${ }^{9-11}$ Despite the apparent benefits of fenestration, drawbacks include the risks of systemic embolization, systemic desaturation, and need for late catheter interventions for fenestration closure. ${ }^{9,12,13}$

The improved hemodynamics and durability, decreased atrial arrhythmias, and relative technical ease of the extracardiac Fontan modification have led to its widespread use for all single-ventricle anatomic subtypes. ${ }^{14,15}$ Greater reliance on the extracardiac technique has facilitated these palliations by the avoidance of cardiac arrest, systemic cooling, and extensive atrial manipulation-all variables that have been shown to have a negative effect on survival. ${ }^{16,17}$ Evolution in perioperative management strategies has further improved outcomes, with advances in anesthetic techniques, perfusion strategies, and postoperative care translating into increased surgical success.

Building on these improvements, our center has developed a highly selective approach to Fontan fenestration, limiting its application only to very high-risk patients. The purpose of this study was to review our outcomes with highly selective Fontan fenestration across all anatomic subtypes.

\section{MATERIALS AND METHODS}

A retrospective analysis of all primary Fontan palliations performed at Texas Children's Hospital between January 1, 2002, and December 31, 


\section{Abbreviations and Acronyms \\ $\mathrm{CPB}=$ cardiopulmonary bypass}

2008 was completed with the permission of the institutional review board of Baylor College of Medicine. There were 226 consecutive Fontan palliations performed, with none excluded. Long-term outcomes were determined from review of patient follow-up records at Texas Children's Hospital and records of referring physicians.

The mean age and weight at operation were $4.3 \pm 3.8$ years and $17.2 \pm$ $9.0 \mathrm{~kg}$, respectively, and $42 \%(\mathrm{n}=95)$ were female. On preoperative evaluation, atrioventricular valve regurgitation was moderate or greater in $4.4 \%$ $(\mathrm{n}=10)$. Mean transpulmonary gradient was $4.1 \pm 2.4 \mathrm{~mm} \mathrm{Hg}$, and mean pulmonary vascular resistance was $1.4 \pm 0.72$ Woods units.

Anatomic subtypes were categorized as single left ventricle $(n=88$, $38.9 \%$ ), single right ventricle including hypoplastic left heart syndrome $(\mathrm{n}=78,34.5 \%)$, common ventricle including unbalanced atrioventricular canal $(n=19,8.4 \%)$, and heterotaxy syndrome $(n=41,18.1 \%)$. Concomitant procedures at the time of Fontan palliation included atrioventricular valve repair $(n=16,7.1 \%)$, aortic reconstruction $(n=2,0.9 \%)$, atrial septectomy $(n=36,15.9 \%)$, pulmonary artery plasty $(n=60,26.5 \%)$, main pulmonary artery division $(\mathrm{n}=11,4.9 \%)$, pacemaker implantation $(\mathrm{n}=7$, $3.1 \%)$, peritoneal dialysis catheter placement $(\mathrm{n}=54,23.9 \%)$, and ligation of Blalock-Taussig shunt $(\mathrm{n}=8,3.5 \%)$.

The surgical technique used for Fontan palliation was either intracardiac lateral tunnel or extracardiac conduit. Lateral tunnel procedures were performed under conditions of cardiopulmonary bypass (CPB) with aortic crossclamping, cardioplegic electromechanical arrest, and moderate systemic cooling. Polytetrafluoroethylene (GORE-TEX; W. L. Gore \& Associates, Inc, Flagstaff, Ariz) patch material was used to baffle the inferior vena cava to the Glenn cavopulmonary connection through the right atrial appendage, and a 2.5- to 4-mm fenestration was created routinely with a punch. Extracardiac palliation was performed under conditions of $\mathrm{CPB}$ with mild hypothermia, without cardiac arrest, and with GORE-TEX conduits ranging in size from 16 to $24 \mathrm{~mm}$, with sizes 18 and 20 predominating. The GORETEX extracardiac conduit was anastomosed to the underside of the pulmonary arteries offset from the Glenn connection and then sewn end-to-end into the divided inferior vena cava.

The CPB circuit (Terumo with RX-15 hollow-fiber oxygenator; Terumo Medical Corporation, Tokyo, Japan) minimized priming volumes $(650 \mathrm{~mL})$, and the pump prime was made as physiologically normal as possible. The pump prime hematocrit was such that the hematocrit during CPB was greater than $30 \%$, and the prime was ultrafiltered (Hemocor; Minntech Corporation, Minneapolis, Minn) as necessary before CPB to ensure physiologic blood chemistry and glucose values. In addition to packed red blood cells, fresh-frozen plasma and $25 \%$ albumin were used for the prime to maintain oncotic pressure. On CPB, continuous zero-balance ultrafiltration was performed for removal of free water and inflammatory mediators. $\mathrm{CPB}$ flows were maintained to achieve a 2.8 cardiac index irrespective of systemic temperature, and the target hematocrit after CPB was greater than $35 \%$. When aortic crossclamping was required, a buffered, crystalloid cardioplegia solution was administered every 20 minutes. Since 2005, a policy of early extubation after Fontan palliation has been in place, with most patients extubated in the operating room.

\section{Statistical Methods}

Descriptive statistics for nominal and numeric data included ratio and mean $\pm \mathrm{SD}$. The $\chi^{2}$ test was used to compare categoric variables, and the Student $t$ test was used to compare continuous variables. Multivariate analysis was performed with regression models (linear for continuous variables and logistic for categoric variables) to study the effects of selected factors on outcome variables. All analyses were conducted with SPSS 13.0 (SPSS Inc, An IBM Company, Chicago, Ill).

\section{RESULTS}

The decreasing use of Fontan fenestration at our center is demonstrated in Table 1. The data presented reflect the total numbers of primary Fontan palliations by anatomic subtype and the percentages of each that were fenestrated. In 2008, only 2 of 32 palliations $(6 \%)$ were fenestrated.

Operative data by Fontan surgical technique are listed in Table 2. Mean aortic crossclamp times in minutes, CPB times in minutes, and lowest systemic temperatures during cooling are listed for extracardiac and lateral tunnel techniques. Intracardiac concomitant procedures were performed in 24 patients receiving extracardiac conduits $(15.3 \%)$, compared with 26 patients receiving lateral tunnels $(37.7 \%)$.

The relative distribution of extracardiac versus lateral tunnel procedures is graphically displayed in Figure 1. As use of the extracardiac technique has increased, overall Fontan fenestration (solid line) has steadily decreased. Currently, fenestration use is highly selective among patients receiving extracardiac tunnels and still common among those receiving lateral tunnels.

Early extubation was defined as occurring in the operating room or at any time during the day of surgery. The rate of early extubation has increased steadily during the study period, across all anatomic subtypes. The rate of early extubation went from $6.3 \%(\mathrm{n}=1)$ in 2002 to $93.8 \%(\mathrm{n}=30)$ in 2008. Early extubation was accomplished in 106 of 157 patients receiving extracardiac conduits $(67.5 \%)$, in contrast to 31 of 69 patients receiving lateral tunnels $(44.9 \%$, $P=.001)$.

Results with decreasing fenestration use are presented in Table 3. Early and late measures of outcome are represented. An early death was defined as one occurring before discharge or within 30 postoperative days. Early death occurred in 3 cases $(1.3 \%)$. One death occurred after an extracardiac Fontan procedure (nonfenestrated) in the setting of dextrocardia, unbalanced atrioventricular canal, and heterotaxy syndrome. The patient had previously undergone bilateral, bidirectional cavopulmonary shunts in the setting of interrupted inferior vena cava (Kawashima). After an initially stable postoperative period, hypotension and ventricular fibrillation developed and were refractory to resuscitation efforts and reopening of the sternum. A second early death occurred after fenestrated extracardiac Fontan procedure in a patient with hypoplastic left heart syndrome and severe reactive airway disease. This patient demonstrated significantly elevated pulmonary vascular resistance postoperatively and ultimately died of multisystem organ failure. The third early death occurred after total cavopulmonary connection including fenestrated lateral tunnel Fontan procedure in the setting of trisomy 21 and unbalanced atrioventricular canal in 
TABLE 1. Fenestration of primary Fontan procedure by anatomic subtype

\begin{tabular}{|c|c|c|c|c|c|c|c|c|}
\hline Anatomy & 2002 & 2003 & 2004 & 2005 & 2006 & 2007 & 2008 & Total \\
\hline Single left ventricle & $5(100 \%)$ & $15(60 \%)$ & $7(14 \%)$ & $14(29 \%)$ & $16(31 \%)$ & $17(12 \%)$ & $14(7 \%)$ & $88(31 \%)$ \\
\hline Single right ventricle & $4(100 \%)$ & $7(71 \%)$ & $13(54 \%)$ & $14(57 \%)$ & $14(43 \%)$ & $16(56 \%)$ & $10(10 \%)$ & $78(51 \%)$ \\
\hline $\begin{array}{l}\text { Hypoplastic left } \\
\text { heart syndrome }\end{array}$ & $2(100 \%)$ & $2(100 \%)$ & $8(62 \%)$ & $8(75 \%)$ & $9(56 \%)$ & $11(36 \%)$ & $7(0 \%)$ & $47(51 \%)$ \\
\hline Common ventricle & $2(50 \%)$ & $4(100 \%)$ & $3(0 \%)$ & $5(100 \%)$ & $1(0 \%)$ & $2(0 \%)$ & $2(0 \%)$ & $19(53 \%)$ \\
\hline Heterotaxy & $5(80 \%)$ & $5(40 \%)$ & $4(100 \%)$ & $7(14 \%)$ & $8(37 \%)$ & $6(67 \%)$ & $6(0 \%)$ & $41(44 \%)$ \\
\hline Total & $16(87 \%)$ & $31(64 \%)$ & $27(44 \%)$ & $40(45 \%)$ & $39(36 \%)$ & $41(37 \%)$ & $32(6 \%)$ & $226(42 \%)$ \\
\hline
\end{tabular}

Data reflect total Fontan procedures performed and percentage with fenestration. Hypoplastic left heart syndrome is listed as a subset of single right ventricle.

a patient who had undergone pulmonary arterial banding. The patient underwent fenestration closure on postoperative day 2 in response to hypoxia. After mechanical pleurodesis for pleural effusion, the patient died of cardiovascular collapse. There have been no early deaths since January 2005. Two late deaths occurred among patients undergoing Fontan procedures before 2004. One death occurred late after transplantation in the setting of hypoplastic left heart syndrome. The second late death, of unclear etiology, occurred after device closure of a fenestrated lateral tunnel Fontan procedure.

Comparison of early outcomes between fenestrated $(\mathrm{n}=95)$ and nonfenestrated $(\mathrm{H}=131)$ Fontan palliations is displayed in Table 4. Presented are the of days of intubation after surgery, intensive care unit stay, hospital stay, and number of days with chest tube in place. In-hospital mortality and early readmission (within 1 month of discharge) rate are also presented. Early outcomes for patients with nonfenestrated procedures were equal to or better than those for patients with fenestrated procedures. As noted previously, patients with fenestrated lateral tunnels predominated in the early years of this study. Furthermore, lateral tunnel procedures were associated with more intracardiac concomitant procedures than were extracardiac procedures.

The median duration of intubation after surgery for patients with fenestration was 1 day (range, 0-33 days); that for patients without fenestration was 0 days (range, 0-5 days). Median intensive care unit stay for patients with fenestration was 3 days (range, 1-33 days); that for patients without fenestration was 2 days (range, $1-22$ days). Median hospital stay for patients with fenestration was 8 days (range, 4-56 days); that for patients without fenestration was 8 days (range, 1-85 days). The median time with chest tubes in place for patients with fenestration was 5 days (range, 1-26 days); that for patients without fenestration was 5.5 days (1-23 days). No patient was discharged home with a chest drain in place.

Readmission within 1 month of discharge was for effusion in 37 patients, for wound infection in 5 patients, and for various, less acute reasons in 12 patients. The readmission rate for effusion among patients with fenestration was $16.8 \%$ (16/95); that among patients without fenestration was $16.0 \%$ (21/131). Discharge oxygen saturation for patients with fenestration averaged $91 \% \pm 4 \%$; that for patients without fenestration averaged $93 \% \pm 5 \%(P=.283)$.

Total hospital charges for patients undergoing Fontan procedures were collected for the years 2004 through 2008 . Mean charges during this period were $\$ 53,492.08 \pm$ $\$ 40,654.57$. Total charges during the years studied did not vary significantly (data not shown). There was a trend $(P=.105)$ toward increased hospital charges for fenestrated $(\$ 58,728.15 \pm \$ 43,502.40)$ versus nonfenestrated Fontan operations $(\$ 50,785.31 \pm \$ 39,017.07)$. Similarly, there was a trend $(P=.115)$ toward increased hospital charges for the lateral tunnel technique $(\$ 62,058.09 \pm \$ 49,572.82)$ versus the extracardiac technique $(\$ 50,615.44 \pm$ $\$ 36,957.40)$. These trends existed despite the differences in era.

Late follow-up was performed by review of clinic, hospital, and referring physician records. Mean length of followup was $2.0 \pm 1.7$ years (range, $0-7$ years). Table 5 shows the late outcomes for patients with and without fenestration, demonstrating that the reintervention rate was significantly higher in the group with fenestration. Indications for early or late reoperation included mediastinal exploration for

TABLE 2. Operative data for primary Fontan Palliations by surgical technique

\begin{tabular}{lccr}
\hline Technique & Crossclamp time (min) & CPB time (min) & Cooling temperature $\left({ }^{\circ} \mathbf{C}\right)$ \\
\hline Extracardiac conduit $(\mathrm{n}=157)$ & $10.9 \pm 26.7^{*}$ & $103.8 \pm 45.5^{*}$ & $31.9 \pm 3.9^{*}$ \\
Concomitant procedure $(\mathrm{n}=24)$ & $49 \pm 40.2$ & $168 \pm 57.5$ & $27 \pm 4.8$ \\
No concomitant procedure $(\mathrm{n}=133)$ & $4 \pm 15.8$ & $92 \pm 31.4$ & $33 \pm 3.0$ \\
Lateral tunnel $(\mathrm{n}=69)$ & $92.3 \pm 33.2$ & $151.4 \pm 36$ & $26.0 \pm 3.3$ \\
Concomitant procedure $(\mathrm{n}=26)$ & $93 \pm 36.6$ & $154 \pm 39.6$ & $27 \pm 2.3$ \\
No concomitant $(\mathrm{n}=43)$ & $92 \pm 31.4$ & $149 \pm 34$ & $25 \pm 3.6$ \\
\hline
\end{tabular}

All data are mean \pm SD. $* P<.001$ versus lateral tunnel. $C P B$, Cardiopulmonary bypass. 


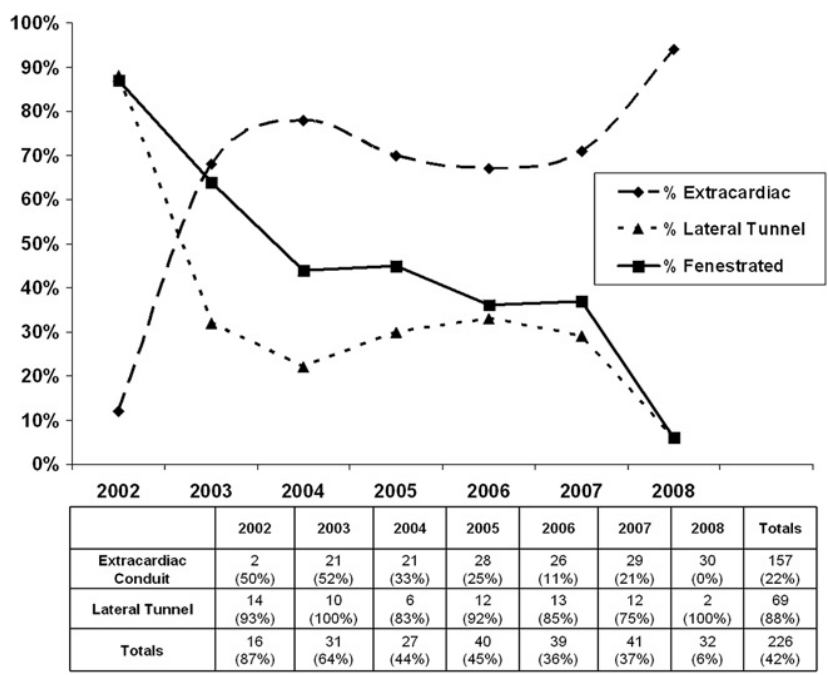

FIGURE 1. Relative use of extracardiac versus lateral tunnel technique and association with overall fenestration. Numbers of patients with percentages of fenestration are shown at bottom.

bleeding in 1 patient, pacemaker placement in 5 patients, pleurodesis or pericardial window in 8 patients, transplantation in 2 patients, and sternal procedures in 3 patients. Indications for early or late postoperative catheter interventions included collateral occlusion in 8 patients, fenestration closure in 13 patients, pacemaker generator change in 3 patients, pulmonary arterial dilation or stenting in 4 patients, diagnostic evaluation in 6 patients, atrial baffle dilation in 1 patient, thoracentesis in 1 patient, atrial puncture in 3 patients, dilation of coarctation in 1 patient, and pericardiocentesis in 1 patient. There were no Fontan circulation takedowns or revisions in the study population.

Late survival in Table 5 accounts for in-hospital deaths and postdischarge deaths. Excluding the 3 in-hospital deaths, late survival was $99.1 \%(221 / 223)$. Late survivals by anatomic subtype were as follows: single left ventricle $100 \%$, single right ventricle $99 \%$, common ventricle $100 \%$, and heterotaxy $97 \%$, irrespective of the use of fenestration (data not shown).

Multivariate regression was performed to identify variables affecting outcomes in patients undergoing Fontan procedures (Table 6). The variables included in the multivariate analysis were ventricular morphology, intracardiac concomitant procedure, Fontan type, Fontan fenestration, degree of systemic cooling, aortic crossclamp time, year of surgery, and early extubation. None of the variables analyzed were predictive of early survival.

Ventricular morphology was identified on multivariate regression as predictive of postoperative stay $(P=.008)$. Single left ventricle was associated with an ICU stay of $2.6 \pm 2.4$ days and a total postoperative stay of $9.0 \pm 9.0$ days. Single right ventricle was associated with an ICU stay of $3.7 \pm 5.0$ days and a total postoperative stay of $11.0 \pm 6.1$ days. Common ventricle was associated with an ICU stay of $5.7 \pm 7.4$ days and a total postoperative stay of $14.2 \pm 15.2$ days. Heterotaxy was associated with an ICU stay of $4.6 \pm 4.5$ days and a total postoperative stay of $12.8 \pm 8.2$ days.

On multivariate analysis, Fontan technique (lateral tunnel) was predictive of longer ICU stay $(P=.022)$ and total postoperative stay $(P=.017)$. Fontan type was not a significant predictor of outcome on univariate analysis. Patients receiving extracardiac conduits had an ICU stay of $3.5 \pm$ 4.2 days and a total postoperative stay of $10.9 \pm 8.4$ days. Similarly, patients undergoing lateral tunnel procedures had an ICU stay of $3.8 \pm 4.8$ days and a total postoperative stay of $10.6 \pm 9.8$ days. Aortic crossclamp duration was predictive of both ICU $(P=0.037)$ and total postoperative $(P=0.016)$ stays on multivariate analysis.

\section{DISCUSSION}

Management of patients with single-ventricle anatomy undergoing Fontan palliation has undergone significant evolution during the last 4 decades, with advances in surgical techniques and perioperative strategies facilitating the achievement of excellent results for these challenging patients. ${ }^{1-5}$ Fontan fenestration has been a traditional mainstay of the surgical approach to improve early outcomes, ${ }^{9-11}$ although we present data here strongly suggesting that fenestration of the Fontan circuit is necessary only in highly selected cases, irrespective of single-ventricle anatomic subtype. Avoidance of fenestration theoretically minimizes the risks of decreased oxygen

TABLE 3. Fenestration use and outcomes

\begin{tabular}{|c|c|c|c|c|c|c|c|c|}
\hline & 2002 & 2003 & 2004 & 2005 & 2006 & 2007 & 2008 & Total \\
\hline Total cases (no.) & 16 & 31 & 27 & 40 & 39 & 41 & 32 & 226 \\
\hline Fenestrated (no.) & $14(87 \%)$ & $20(64 \%)$ & $12(44 \%)$ & $18(45 \%)$ & $14(36 \%)$ & $15(37 \%)$ & $2(6 \%)$ & $95(42 \%)$ \\
\hline ICU stay (d, mean \pm SD) & $4.3 \pm 4.8$ & $3 \pm 1.8$ & $4.3 \pm 6.6$ & $4.6 \pm 6.5$ & $3.2 \pm 3.1$ & $3.6 \pm 3.4$ & $2.5 \pm 2.3$ & $3.6 \pm 4.4$ \\
\hline Hospital stay $(\mathrm{d}$, mean $\pm \mathrm{SD})$ & $11.8 \pm 9.8$ & $8.4 \pm 4$ & $11.8 \pm 7.7$ & $12.7 \pm 12.1$ & $10 \pm 6.1$ & $11.4 \pm 12.3$ & $9.3 \pm 2.8$ & $10.8 \pm 8.8$ \\
\hline $\begin{array}{l}\text { Discharge oxygen saturation } \\
\quad(\%, \text { mean } \pm \mathrm{SD})\end{array}$ & $88 \pm 6.7$ & $91.3 \pm 4.6$ & $90.7 \pm 3.4$ & $93.1 \pm 4.6$ & $92.7 \pm 3.5$ & $93 \pm 4.2$ & $92.4 \pm 4.1$ & $92 \pm 4.5$ \\
\hline Early deaths (no.) & $0(0 \%)$ & $1(3.2 \%)$ & $1(3.7 \%)$ & $1(2.5 \%)$ & $0(0 \%)$ & $0(0 \%)$ & $0(0 \%)$ & $3(1.3 \%)$ \\
\hline Late deaths (no.) & $1(6.2 \%)$ & $1(3.2 \%)$ & $0(0 \%)$ & $0(0 \%)$ & $0(0 \%)$ & $0(0 \%)$ & $0(0 \%)$ & $2(1 \%)$ \\
\hline
\end{tabular}

$I C U$, Intensive care unit. 
TABLE 4. Comparison of early outcomes after fenestrated and nonfenestrated Fontan procedures

\begin{tabular}{lccc}
\hline & Fenestrated & Nonfenestrated & $\boldsymbol{P}$ value \\
\hline Intubation $(\mathrm{d}$, mean $\pm \mathrm{SD})$ & $1.4 \pm 4.3$ & $0 \pm 0.7$ & .002 \\
ICU stay $(\mathrm{d}$, mean $\pm \mathrm{SD})$ & $4.3 \pm 5.6$ & $3.1 \pm 3.3$ & .039 \\
Hospital stay (d, mean $\pm \mathrm{SD})$ & $11.8 \pm 9.6$ & $10.9 \pm 8.3$ & .14 \\
Chest tube (d, mean $\pm \mathrm{SD})$ & $6.0 \pm 3.5$ & $6.6 \pm 4.5$ & .025 \\
In-hospital deaths (no.) & $2(2 \%)$ & $1(1 \%)$ & .38 \\
Early readmissions (no.) & $22(23 \%)$ & $32(24 \%)$ & .477 \\
\hline
\end{tabular}

*Early readmissions were those within 30 days of discharge. ICU, Intensive care unit.

saturation, systemic embolization, arrhythmias and heart block, and need for late catheter interventions. ${ }^{9,12,13}$

The early and late outcomes presented here with a strategy of highly selective Fontan fenestration are encouraging and possibly even better than those after routine fenestrated Fontan procedures. Other groups who have adopted this surgical approach also report favorable outcomes. ${ }^{18-20}$ Fenestration is reserved for the patients at highest risk, a group that may include patients undergoing significant concomitant procedures or single-lung Fontan palliation, those with elevated pulmonary vascular resistance or transpulmonary gradient, those with significant atrioventricular valve regurgitation or poor ventricular function, and those with intracardiac anatomy not amenable to extracardiac conduit. ${ }^{8,9,21}$

Advances in all aspects of patient management have collectively enabled the results presented here for nonfenestrated procedures. With improved outcomes after first- and second-stage single-ventricle palliation, better candidates for completion Fontan procedures are referred for surgery. Improved medical management and interstage surveillance are also likely to have contributed. The population undergoing Fontan procedures in the experience reported here is somewhat older than that reported by others, which may further contribute to a decreased need for fenestration. ${ }^{22}$

At our institution, the association between increased use of the extracardiac technique and decreased fenestration is clear. Extracardiac conduits for completion of total cavopulmonary anastomosis minimize the need for cardiac arrest, prolonged $\mathrm{CPB}$, extensive atrial manipulation, and systemic

TABLE 5. Late outcomes after fenestrated and nonfenestrated Fontan procedures

\begin{tabular}{lcccc}
\hline & Fenestrated & Nonfenestrated & Total & $\boldsymbol{P}$ value \\
\hline Reoperations & $12(12.6 \%)$ & $7(5.2 \%)$ & $19(8.4 \%)$ & .04 \\
Catheterization & $28(29.5 \%)$ & $8(6.1 \%)$ & $36(15.9 \%)$ & .001 \\
Late survival & $92(96.8 \%)$ & $129(98.5 \%)$ & $221(97.8 \%)$ & $>.10$ \\
Stroke & $0(0 \%)$ & $0(0 \%)$ & $0(0 \%)$ & $>.10$ \\
Transplant & $1(1.1 \%)$ & $1(0.8 \%)$ & $2(0.9 \%)$ & $>.10$ \\
Fontan takedown & $0(0 \%)$ & $0(0 \%)$ & $0(0 \%)$ & $>.10$ \\
$\quad$ or revision & & & & \\
All data represent numbers and percentages of patients. Mean follow-up $2.0 \pm 1.7$ \\
years.
\end{tabular}

TABLE 6. Multivariate analysis of outcome predictors ( $P$ values $)$

\begin{tabular}{lccc}
\hline & \multicolumn{2}{c}{ Stay (d) } & \\
\cline { 2 - 3 } & ICU & Postoperative & Operative survival \\
\hline Ventricular morphology & .068 & .008 & .932 \\
Concomitant procedure* & .538 & .284 & .593 \\
Fontan type & .022 & .017 & .181 \\
Fontan fenestration & .143 & .407 & .807 \\
Lowest temperature & .612 & .932 & .343 \\
Crossclamp time & .037 & .016 & .309 \\
Year of Fontan & .656 & .571 & .998 \\
Early extubation $(<24 \mathrm{~h})$ & .078 & .516 & .995 \\
\hline *Fontan procedure with concomitant intracardiac procedure. $I C U$ Intensive care unit.
\end{tabular}

cooling. ${ }^{17,18}$ These advantages likely decrease the impact of the surgical procedure on cardiopulmonary physiology, thereby limiting the need for fenestration to support cardiac output. With increased use of nonfenestrated extracardiac conduits, measures of early morbidity such as hospital stay and need for chest drains are not increased. Even for patients who require intracardiac or other significant concomitant procedures, an extracardiac Fontan procedure lessens the myocardial ischemic time and may translate into improved outcome. Furthermore, less systemic cooling lower the impact on pulmonary physiology and thus decrease the need for fenestration. Even for patients with challenging intracardiac, caval, or pulmonary venous anatomy, the extracardiac conduit has been applied with excellent results. ${ }^{5,7,18,23}$ Groups performing the Fontan procedure in a younger patient population and with standard use of the intracardiac tunnel technique and circulatory arrest routinely fenestrate. ${ }^{22}$ In the current era, whether routine fenestration in the lateral tunnel Fontan procedure is indicated still remains to be evaluated.

Careful management of perfusion and anesthesia during Fontan palliation likely contributes to the decreased need for fenestration. At Texas Children's Hospital, our perfusion strategy emphasizes a physiologic pump prime, maintenance of full CPB flow irrespective of systemic temperature, aggressive ultrafiltration for removal of free water and inflammatory mediators, and careful attention to myocardial protection. Having said this, other groups have advocated extracardiac Fontan procedures without the use of CPB and reported encouraging outcomes. ${ }^{24}$ Our approach has been to use CPB for all Fontan palliations, because we believe that $\mathrm{CPB}$ facilitates a controlled, comprehensive procedure without compromising outcomes.

Key elements of anesthetic management include the judicious administration of opioids and sedation, the use of regional anesthetic techniques, and a policy of early extubation. ${ }^{25}$ Fontan physiology is impaired with positive-pressure ventilation. $^{26}$ Our approach has therefore been aimed at extubation in the operating room or very soon thereafter. ${ }^{25}$ The addition of dexmedetomidine to achieve sedation without respiratory depression has further facilitated this 
strategy. ${ }^{27}$ Immediate postoperative management is complementary, with emphasis on minimizing both volume administration and excessive sedation. Long-term anticoagulation management is typically with aspirin alone. Other medical management includes furosemide and afterload reduction, as indicated.

In summary, we present our experience with highly selective use of fenestration during Fontan palliation. The data support a nonfenestrated, extracardiac conduit technique for Fontan palliation, irrespective of anatomic subtype, with fenestration reserved for only the patients at highest risk. Relying on improvements in all areas of medical and surgical management, this strategy results in equal or better surgical outcomes and fewer reinterventions. Further longitudinal follow-up of this patient population, including functional outcomes, will benefit the assessment of this approach for patients with single-ventricle anatomy.

We thank all our team members at Texas Children's Hospital for their tireless dedication to and care of these patients. Without them, these results for our patients undergoing Fontan palliation would not be possible.

\section{References}

1. Fontan F, Baudet E. Surgical repair of tricuspid atresia. Thorax. 1971;26:240-8.

2. deLeval MR, Kilner P, Gewillig M, Bull C. Total cavopulmonary connection: a logical alternative to atriopulmonary connection for complex Fontan operations. Experimental studies and early clinical experience. J Thorac Cardiovasc Surg. 1988;96:682-95.

3. Amodeo A, Galletti L, Marianeschi S, Picardo S, Giannico S, DiRenzi P, et al. Extracardiac Fontan operation for complex cardiac anomalies: seven years' experience. J Thorac Cardiovasc Surg. 1997;114:1020-30.

4. d'Udekem Y, Iyengar AJ, Cochrane AD, Grigg LE, Ramsay JM, Wheaton GR, et al. The Fontan procedure: contemporary techniques have improved longterm outcomes. Circulation. 2007;116(11 Suppl):I157-64.

5. Bando K, Turrentine MW, Park HJ, Sharp TG, Scavo V, Brown JW. Evolution of the Fontan procedure in a single center. Ann Thorac Surg. 2000;69:1873-9.

6. Gentles TL, Mayer JE Jr, Gauvreau K, Newburger JW, Lock JE, Kupferschmid JP, et al. Fontan operation in five hundred consecutive patients: factors influencing early and late outcomes. J Thorac Cardiovasc Surg. 1997; 114:376-91.

7. Anderson PA, Sleeper LA, Mahony L, Colan SD, Atz AM, Breitbart RE, et al. Contemporary outcomes after the Fontan procedure. J Am Coll Cardiol. 2008; 52:85-98.

8. Hosein RB, Clarke AJ, McGuirk SP, Griselli M, Stumper O, DeGiovanni JV, et al. Factors influencing early and late outcome following the Fontan procedure in the current era. The 'Two Commandments?'. Eur J Cardiothorac Surg. 2007; 31:344-52.

9. Bridges ND, Lock JE, Castaneda AR. Baffle fenestration with subsequent transcatheter closure: modification of the Fontan operation for patients at increased risk. Circulation. 1990;82:1681-9.

10. Ono M, Boethig D, Goerler H, Lange M, Westhoff-Bleck M, Breymann T. Clinical outcome of patients 20 years after Fontan operation - effect of fenestration on late morbidity. Eur J Cardiothorac Surg. 2006;30:923-9.

11. Lemler MS, Scott WA, Leonard SR, Stromberg D, Ramaciotti C. Fenestration improves clinical outcome of the Fontan procedure: a prospective, randomized study. Circulation. 2002;105:207-12.

12. Monagle P, Karl TR. Thromboembolic problems after the Fontan operation. Semin Thorac Cardiovasc Surg Pediatr Card Surg Annu. 2002;5:36-47.

13. Kaulitz R, Ziemer G, Paul T, Peuster M, Bertram H, Hausdorf G. Fontan-type procedures: residual lesions and late interventions. Ann Thorac Surg. 2002;74: 778-85.

14. Azakie A, McCrindle BW, Van Arsdell G, Benson LN, Coles G, Hamilton R, et al. Extracardiac conduit versus lateral tunnel cavopulmonary connections at a single institution: impact on outcomes. J Thorac Cardiovasc Surg. 2001;122: 1219-28.

15. deLeval MR, Dubini G, Migliavacca F, Jalali H, Camporini G, Redington A, et al. Use of computational fluid dynamics in the design of surgical procedures: application to the study of competitive flows in cavo-pulmonary connections. J Thorac Cardiovasc Surg. 1996;111:502-13.

16. Bove EL, Lloyd TR. Staged reconstruction for hypoplastic left heart syndrome. Contemporary results. Ann Surg. 1996;224:387-95.

17. Tam VK, Miller BE, Murphy K. Modified Fontan without use of cardiopulmonary bypass. Ann Thorac Surg. 1999;68:1698-704.

18. Thompson LD, Petrossian E, McElhinney DB, Abrikosova NA, Moore P, Reddy VM, et al. Is it necessary to routinely fenestrate an extracardiac Fontan? J Am Coll Cardiol. 1999;34:539-44.

19. Hsu DT, Quaegebeur JM, Ing FF, Selber EJ, Lamour JM, Gersony WM. Outcome after the single-stage, nonfenestrated Fontan procedure. Circulation. 1997;96(9 Suppl):II335-40.

20. Harada Y, Uchita S, Sakamoto T, Kimura M, Umezu K, Takigiku K, et al. Do we need fenestration when performing two-staged total cavopulmonary connection using an extracardiac conduit? Interact Cardiovasc Thorac Surg. 2009;9:50-4.

21. Choussat A, Fontan F, Besse P. Selection criteria for Fontan's procedure. In: Anderson RH, Shinebourne EA, eds. Pediatric cardiology. Edinburgh: Churchill Livingstone; 1978. p.559-66.

22. Meyer DB, Zamora G, Wernovsky G, Ittenbach RF, Gallagher PR, Tabbut S, et al. Outcomes of the Fontan procedure using cardiopulmonary bypass with aortic cross-clamping. Ann Thorac Surg. 2006;82:1611-8.

23. Hirsch JC, Goldberg CG, Bove EL, Salehian S, Lee T, Ohye RG, et al. Fontan operation in the current era: a 15-year single-institution experience. Ann Surg. 2008;248:402-10.

24. Petrossian E, Reddy VM, Collins KK, Culbertson CB, MacDonald MJ, Lamberti JJ, et al. The extracardiac conduit Fontan operation using minimal approach extracorporeal circulation: early and midterm outcomes. J Thorac Cardiovasc Surg. 2006; 132:1054-63.

25. Morales DL, Carberry KE, Heinle JS, McKenzie ED, Fraser CD Jr, Diaz LK. Extubation in the operating room after Fontan's procedure: effect on practice and outcomes. Ann Thorac Surg. 2008;86:576-81.

26. Chin AJ, Fogel MA. Atrial/vena caval/pulmonary venous operations, In: Noninvasive imaging of congenital heart disease: before and after surgical reconstruction. Armonk (NY): Futura Publishing; 1994.

27. Mukhtar AM, Obayah EM, Hassona AM. The use of dexmedetomidine in pediatric cardiac surgery. Anesth Analg. 2006;103:52-6.

\section{Discussion}

Dr Scott M. Bradley (Charleston, SC). You have nicely traced the evolution of your group's strategy through the last 7 years to one that now includes use of an extracardiac conduit, with the use of $\mathrm{CPB}$, minimal use of aortic crossclamping, and decreasing in use of fenestrations (only 6\% of patients in the last year). You have shown without question that this strategy can be used with good outcomes, looking at all the usual outcome measures that are examined for Fontan procedures. Both the use of this strategy and your outcomes are in line with what has been described in previous publications, for example, those from the Stanford/University of California San Francisco experience and from the Los Angeles Children's Hospital experience.

I have several questions. The first has to do with the duration of pleural effusion drainage in your study, which ran about 6 days. This was discussed in the debate yesterday, but there is certainly a great deal of variation in the literature; some of this variation is undoubtedly related to differences in management protocols for chest tubes after Fontan procedures. Could you elaborate on your group's approach to postoperative management of chest tubes? Specifically, when do you decide to remove chest tubes? Do you have any consistent approach to the use of medications to try to cut down on effusion drainage, such as angiotensin-converting enzyme inhibitors and diuretics? 
Dr Salazar. Our strategy for decreasing chest tube output begins with aggressive ultrafiltration in the operating room, minimization of fluid administration in the postoperative period, and a policy of early extubation. With regard to timing of chest tube removal, all of us at Texas Children's Hospital are conservative and prefer to leave the tubes in until minimal output has been demonstrated. Diuretic administration is routine, preferring to see a mild increase in blood urea nitrogen as a result of our diuretic management. Furthermore, patients are fluid restricted to half or three-quarters maintenance total fluid intake for at least the first week after surgery.

Dr Bradley. Do you have any specific amount of chest tube drainage that you use to decide when to take the chest tubes out?

Dr Salazar. I prefer to tailor that decision to the specific patient, taking into account the clinical situation. As a general rule, we do not remove drains until output has decreased below $1 \mathrm{~mL} /(\mathrm{kg} \cdot \mathrm{d})$.

Dr Bradley. It appeared that approximately a quarter of your patients were readmitted. I assume that some of these were for treatment of effusions?

Dr Salazar. Correct. Readmission was defined as occurring any time within 1 month of discharge, to capture late readmissions. More than half of those readmissions were for pleural effusion management. Interestingly, the percentages of patients requiring readmission for effusion in the fenestrated and nonfenestrated groups were equivalent.

Dr Bradley. If you were to include that period in the duration of pleural effusion drainage and the stay data, both of those pieces of information would probably look a bit different. That may be something you would want to consider putting in the article.

You now fenestrate very selectively. How do you decide in which cases to fenestrate? Do you decide preoperatively or in the operating room, and what specific criteria do you look at?

Dr Salazar. The decision to fenestrate is a clinical judgment made in the operating room. Patients known to be at higher risk, such as those receiving single-lung Fontan palliation, those with severe atrioventricular valve regurgitation, and those with high pulmonary vascular resistance are most likely to have fenestration.

The conduct of the operation also influences the fenestration decision. If a significant period of cardiac arrest or even circulatory arrest is required (although this is rare), then fenestration is more likely. Fenestration is used to improve the early outcomes after the Fontan operation. If preoperative and intraoperative variables suggest a risk compromising the patient's early postoperative course, then we would fenestrate. Ultimately, it is a clinical judgment that is based on the experience that fenestration is usually not necessary.

Dr Bradley. So it sounds like a general gestalt of the patient's characteristics and how things are going in the operating room, as opposed to specific hemodynamic criteria?

Dr Salazar. Ultimately, I agree.

Dr Bradley. Fair enough. Finally, I think it's a bit more difficult to fenestrate reliably extracardiac conduits than intra-atrial lateral tunnels. Can you comment on your group's technique?

Dr Salazar. Our technique is to fenestrate extracardiac conduits with the heart beating. We use a partial occlusion clamp on the GORE-TEX conduit and atrium. An aortic punch is used for the GORE-TEX conduit fenestration, although the depth of sutures on the atrial side can influence the effective size of the fenestration.
Dr Bradley. So it's side-to-side between the atrium and the outside of the GORE-TEX graft, as opposed to using a second tube graft between the two?

Dr Salazar. That's correct.

Dr Bradley. I think you have some information in your article about the reliability of fenestration, specifically looking at postoperative systemic oxygen saturation. Can you just comment on the postoperative oxygen saturation difference between the patients with and without fenestration?

Dr Salazar. Most of the patients with fenestrated Fontan procedures underwent the lateral tunnel technique. The lateral tunnel baffle was fenestrated with an aortic punch, typically ranging in size from 3 to $4 \mathrm{~mm}$.

Interestingly, the discharge oxygen saturations were similar in the patients with and without fenestration. This may reflect a less ill patient population referred for Fontan palliation relative to 10 years ago. Decreasing preoperative transpulmonary gradients and pulmonary vascular resistance, coupled with operations that minimize the negative physiologic impact of surgery, have likely led to less shunting across the fenestration.

Dr Bradley. So the numbers were, say, $92 \%$ versus $94 \%$ ?

Dr Salazar. Average discharge saturations were $91 \%$ with fenestration and $93 \%$ without fenestration.

Dr Francois Lacour-Gayet (Aurora, Colo). I enjoyed your presentation. I understand that the rule now is no fenestration. Do you believe that there is an exception for altitude? In Denver, we fenestrate all our Fontan procedures and would not dare not to fenestrate. Can you elaborate on this and perhaps give us a limit, an altitude at which you would consider not to fenestrate?

Dr Salazar. I appreciate your question, Dr Lacour-Gayet. Not having lived or operated in Denver, I won't presume to make a general recommendation for the city. Having said this, the decreased partial pressure of oxygen at altitude likely translates into some increase in pulmonary vascular resistance and reactivity. I would proceed cautiously and base recommendations on clinical experience.

Dr James S. Tweddell (Milwaukee, Wis). You were talking about aggressive use of diuretics. What specific diuretics are you using?

Dr Salazar. Most of the time, we use furosemide alone for postoperative diuresis. Our dose typically is $1 \mathrm{mg} / \mathrm{kg}$, administered 2 or 3 times a day. Some may not consider that aggressive, but that is what I'm talking about.

Dr Tweddell. Did you ever consider using spironolactone in addition?

Dr Salazar. We have used spironolactone in addition to furosemide for those few patients with prolonged chest tube drainage.

Dr Frank L. Hanley (Stanford, Calif). When you do your sideto-side fenestration with the punch and then you put the atrium against that hole, do you put your sutures directly into the edge of the punch when you're actually making the surgical connection?

Dr Salazar. You mean to the inside?

Dr Hanley. When you're bringing the atrium to the GORETEX-I presume you use GORE-TEX?

Dr Salazar. Yes.

Dr Hanley. Are the sutures that go through the GORE-TEX going through the edge of the hole? I mean, are the sutures going into the actual hole of the 4-mm punch? 
Dr Salazar. Yes, I typically take thin bites of atrium and GORETEX to avoid compromising the effective size of the fenestration.

Dr Hanley. If I could just make a suggestion?

Dr Salazar. Please do.

Dr Hanley. We used to do that, too. When you do that, if you pick up too much of the atrium or the atrium is bulky, which is not uncommon in a single ventricle, you will get a tunnel effect to the fenestration because the atrial tissues impinge on the punch hole, making the size of the hole unreliable. We prefer to put a large, side-biting clamp on the GORE-TEX tube and then make the 4-mm punch inside the relatively large surface area of GORE-TEX sequestered by the clamp. We put another large, side-biting clamp on the atrium, sequestering a relatively large surface area of atrium as well. Then we make a long atriotomy, maybe $2 \mathrm{~cm}$, and sew the cut edge of the atrium onto the sequestered surface of the GORETEX graft, at least half a centimeter if not more away from the edge of the punch hole, onto the flat surface of the GORE-TEX. So in the end, if you were sitting in the atrium and looking at that atrial incision, you would see a $1.5-\mathrm{cm}$ circle or oval of GORETEX with a 4-mm punch in the middle of it. It is an undistorted true 2-dimensional fenestration.

Dr Salazar. You have not had any problems with clots forming in that area?

Dr Hanley. Not to our knowledge.

Dr Tweddell. I would just say that we use exactly the same technique. And actually I use the inferior vena cava, or the atrial end of the inferior vena cava. You can use that to sew around the fenestration, just as Dr Hanley suggested, and we have not seen any consequences. It gives you a reliable fenestration.

Dr Salazar. Thank you, and your point is well taken. The good news is that, excepting the possibilities at high altitude, fenestration of extracardiac conduits is rarely necessary. 